\title{
1 Acoustogenetic Control of CAR T Cells via Focused Ultrasound
}

3 Yiqian $\mathrm{Wu}^{1}$, Yahan Liu ${ }^{2,3}$, Ziliang Huang ${ }^{2}$, Xin Wang ${ }^{1}$, Zhen Jin ${ }^{4}$, Jiayi $\mathrm{Li}^{1}$, Praopim Limsakul ${ }^{5}$,

4 Linshan Zhu ${ }^{1}$, Molly Allen ${ }^{1}$, Yijia Pan $^{1}$, Robert Bussell ${ }^{6}$, Aaron Jacobson ${ }^{6}$, Thomas Liu ${ }^{6}$, Shu

5 Chien $^{1,2, *}$, Yingxiao Wang ${ }^{1,2, *}$

6

$7 \quad{ }^{1}$ Department of Bioengineering, University of California, San Diego, La Jolla, CA, USA.

$8{ }^{2}$ Institute of Engineering in Medicine, University of California, San Diego, La Jolla, CA, USA.

$9 \quad{ }^{3}$ Institute of Cardiovascular Sciences, Peking University Health Science Center, Beijing, China.

$10{ }^{4}$ Ruijin Hospital, Shanghai, China.

$11 \quad{ }^{5}$ Department of Physics, Prince of Songkla University, Songkhla, Thailand.

$12{ }^{6}$ Center for Functional MRI, University of California, San Diego, La Jolla, CA, USA.

$13{ }^{*}$ Correspondence to: Email: yiw015@eng.ucsd.edu (Y. Wang); shuchien@ucsd.edu (S. C.) 


\section{Abstract}

15 Optogenetics can control specific molecular events in living systems, but the penetration depth of

16 light is typically limited at hundreds of micrometers. Focused ultrasound (FUS), on the other

17 hand, can deliver energy safely and noninvasively into tissues at depths of centimeters. Here we

18 have developed an acoustogenetic approach using short-pulsed FUS to remotely and directly

19 control the genetics and cellular functions of engineered mammalian cells for therapeutic

20 purposes. We applied this acoustogenetic approach to control chimeric antigen receptor (CAR) T

21 cells with high spatiotemporal precision, aiming to mitigate the potentially lethal "on-target off-

22 tumor" effects of CAR T cell therapy. We first verified the controllability of our acoustogenetic

23 CAR T cells in recognizing and killing tumor cells in vitro, and then applied this approach in

24 vivo to suppress tumor growth of both lymphoma and prostate cancers. The results indicate that

25 FUS-based acoustogenetics can allow the noninvasive and remote activation, without any

26 exogenous cofactor, of different types of CAR T cells for cancer therapeutics. 
27 Optogenetics enables the control of specific molecular events and cellular functions in living

28 systems with high spatiotemporal resolutions. However, optogenetics cannot reach deep tissues,

29 with the penetration depth of light typically limited at micrometer to millimeter scales (1).

30 Ultrasound can be focused to deliver mechanical energy safely and noninvasively into small

31 volumes of tissue deep inside the body up to tens of centimeters (1). The rapidly oscillating

32 pressure of focused ultrasound (FUS) waves and the resultant cycles of mechanical

33 loading/unloading can lead to local heat generation in biological tissues. Aided by Magnetic

34 Resonance Imaging (MRI) thermometry, FUS has been widely applied to clinically ablate

35 tumors, and control drug delivery, vasodilation, neuromodulation (2), and transgene expression

36 (3-5). Transcription factors and genetic circuits have also been engineered to convert the FUS-

37 generated heat into genetic regulation to control microbial systems in vivo (6). However, there is

38 a lack of general methods using FUS to control mammalian cell functions in vivo for therapeutic

39 applications.

41 Chimeric antigen receptor (CAR) $\mathrm{T}$ cell therapy, where $\mathrm{T}$ cells are genetically programmed with

42 redirected specificity against malignant cells, is becoming a paradigm-shifting approach for

43 cancer treatment, especially for blood cancers (7). However, major challenges remain for solid

44 tumors before CAR-based immunotherapy can be widely adopted. For instance, the non-specific

45 targeting of the CAR T cells against normal tissues (on-target off-tumor toxicities) can be life-

46 threatening: off-tumor toxicities against the lung, the brain, and the heart have caused multiple

47 cases of deaths (7-10). Immunosuppressive corticosteroid therapy and suicide gene engineering

48 are relatively effective in suppressing off-tumor toxicities and related cytokine release syndrome

49 (CRS), but they fail to discriminate between beneficial T cell functions and toxic side effects 
(11-13). Synthetic biology and genetic circuits have been used to enhance specificity and reduce

51 off-tumor toxicity by creating chemically inducible dimerization of split CARs, inhibitory CARs

52 (iCARs), and SynNotch to control CAR activation $(8,14-18)$. However, given the extensive

53 overlaps of antigens between solid tumors and normal tissues, especially those under conditions

54 of tissue injury/inflammation (19), it remains very difficult to identify ideal antigens and their

55 combinations to differentiate tumors from normal tissues. There is hence an urgent need for a

56 high-precision control of CAR-T cells to confine the activation at local sites of solid tumors.

57 Recently, we demonstrated that ultrasound signals can be amplified by microbubbles coupled to

58 cells engineered with the mechanosensor Piezol to precisely control CAR T cell activations (20).

59 However, the presence of microbubbles as cofactors limits the application of this system in vivo.

60 Here, we have engineered a new class of inducible CAR T cells that can be remotely and directly

61 controlled by FUS without any exogenous cofactor. We show that short-pulsed FUS stimulation

62 can activate the engineered $\mathrm{T}$ cells at the desired time and location to suppress tumor growth in

63 vivo.

\section{Results}

\section{Heat-induced reporter gene activation}

66 We propose to genetically engineer T cells with inducible CAR cassettes that can be remotely

67 and directly activated, without any exogenous cofactor, by MRI-guided FUS at local tumor sites

68 for recognizing and eradicating the tumor cells (Fig. 1a).

69

70 We first tested the inducible activation of a reporter eGFP under the control of the heat-shock-

71 protein promoter (Hsp). We assembled a dual-promoter reporter construct containing the Hsp- 
72 driven eGFP and a constitutive PGK-driven mCherry (Fig. 1b). HEK 293T cells infected with

73 the reporter lentivirus (fig. S1a) were heated at $43^{\circ} \mathrm{C}$ for $15 \mathrm{~min}$. Real-time fluorescence imaging

74 revealed that the heat-induced eGFP expression started as early as $2 \mathrm{hr}$ after heat shock (HS) and

75 persisted throughout the course of observation (Fig. 1c and Movie S1). Quantitative tracking of

76 the dynamics of heat-induced eGFP expression by flow cytometry showed that $97 \%$ of the cells

77 expressed eGFP at $6 \mathrm{hr}$ post HS, and the percentage increased to $99 \%$ at $12 \mathrm{hr}$ and remained

78 stable for 2 days, while the mean fluorescence intensity peaked at $12 \mathrm{hr}$ followed by a steady

79 decrease (Fig. 1d). We then investigated the inducible effect of HS in primary human T cells

80 hosting the dual-promoter eGFP reporter (fig. S1b). A 15-min HS induced a strong eGFP

81 expression in $92.9 \%$ of the engineered T cells, in contrast to a background of $3.9 \%$ in control

82 cells without HS (Fig. 1, e and f). The mean fluorescence intensity of the eGFP+ cells in the HS

83 group was 10-fold of that in the control group without HS (Fig. 1g).

\section{Heat-induced CAR expression and its functionality in Jurkat and primary human T cells}

85 In order to convert the transient heat stimulation to a sustained gene activation and cellular

86 functions for therapeutic actions, we integrated the Cre-lox gene switch into the inducible

87 system. The design is composed of two constructs, one containing the Hsp-driven Cre

88 recombinase and the PGK-driven membrane c-Myc tag for cell sorting ("inducible Cre", Fig.

89 2a), and the other containing a lox-flanked "ZsGreen-STOP" sequence between a PGK promoter

90 and an anti-CD19 CAR ("lox-stop CAR reporter", Fig. 2a). As such, the excision of the "STOP"

91 cassette mediated by the transient heat-induced Cre can cause a switch from ZsGreen to

92 sustained CD19CAR production. 
94 We first tested this heat-inducible gene switch system in Jurkat T cell lines (fig. S2a). A 15-min

95 HS induced CAR expression in $76.6 \%$ of the cells when measured $24 \mathrm{hr}$ after HS (Day 1), in contrast to a basal value of $14.0 \%$ in control cells without HS and a minimal leakage of $0.6 \%$ in

97 cells infected with the lox-stop CAR reporter alone (Fig. 2b). The heat-induced CAR expression

98 remained stable when measured 6 days after HS (Day 6, Fig. 2b). We further examined the

99 functionality of the induced CD19CAR in engineered cells (Fig. 2c). Engineered Jurkat cells

100 with (HS) or without (CT, control) a 15-min HS were co-cultured with CD19-expressing Nalm-6 tumor cells for $24 \mathrm{hr}$. Quantification of the expression level of CD69 (an early T cell activation marker) revealed a $73.4 \%$ CD69+ cell population in the engineered Jurkat cells in the HS group, in contrast to a $11.9 \%$ in the control group (Fig. 2, $\mathrm{d}$ and e). These results indicate that the HS-

104 induced CD19CAR is efficient for functional changes in engineered Jurkat T cells.

We then examined our system in primary human T cells (Fig. 2, a and c; fig. S2b). CAR antibody staining showed that a 15-min HS induced CAR expression in $29 \%$ of the T cells, in contrast to $1.9 \%$ in control cells without HS (Fig. 2f). The heat-inducible CAR T cells were then co-cultured with firefly luciferase (Fluc)-expressing Nalm-6 cells at different effector-to-target (E:T) ratios for cytotoxicity assays. The luminescence of the remaining Nalm-6 cells was

111 quantified after 24-hr co-culture. The heat-stimulated T cells (HS) demonstrated increased 112 cytotoxicity with increased E:T ratio, with the largest difference in cytotoxicity between the HS 113 and control (CT) T cells observed at E:T $=1: 5$, eliminating $82.9 \%$ and $29.3 \%$ of the target tumor 114 cells, respectively (Fig. 2g). The heat-stimulated CAR T cells also released significantly higher 115 concentrations of cytokines (IFN- $\gamma$ and IL-2) than the control cells when co-cultured with Nalm- 
6 cells (Fig. 2, h and i), verifying the functional capability gained with the HS-induced CAR T

117 cells.

119 While the continuous 15 min HS could lead to strong gene inductions (Figs. 1 and 2), it may cause toxicity to cells (21). We hence investigated the effect of different HS patterns in primary human T cells (fig. S3). Our results showed that longer HS resulted in more cell death; however, pulsed HS was able to alleviate this toxicity while achieving induction levels comparable to that in response to continuous HS with the same total heating time (fig. S3). In particular, a pulsed HS with $50 \%$ duty cycle and a total heating time of 15 min (fig. S3a, Pattern 2) caused a strong induction of eGFP expression in $91.4 \%$ of the engineered $\mathrm{T}$ cells, with a minimal toxicity as evidenced by the $92.2 \%$ cell viability measured $24 \mathrm{hr}$ after HS (fig. S3, b to d). Therefore, we applied this HS pattern (fig. S3a, Pattern 2) for in vivo therapeutic studies.

\section{MRI-guided FUS-induced gene activation in phantom and in vivo}

MRI-guided FUS enables the delivery of thermal energy in vivo at confined local regions with high spatiotemporal resolutions (3, 4). We integrated an MRI-guided FUS system (Image Guided

Therapy) with a 7T MRI as described in Methods. An annular array transducer is placed above the target region of the object to be heated (phantoms or small animals) in the MRI bore. MR images are acquired and transferred to Thermoguide software to calculate the temperature of the target region in real-time, which is fed back to the PID controller to automatically regulate the output power of the FUS generator, maintaining the temperature of the target region at the

136 desired level (Fig. 3a, fig. S4). 
We transduced Nalm-6 cells with a lentiviral dual-luciferase reporter containing inducible Fluc and constitutive Rluc (Hsp-Fluc-PGK-Rluc-mCherry; Rluc, Renilla luciferase; Fig. 3b) and embedded them in a tofu phantom approximately $7 \mathrm{~mm}$ deep from the top surface (Fig. $3 \mathrm{c}$ and Methods). We then focused the ultrasound on the embedded cells by changing the focal distance in the $\mathrm{z}$ direction. Three pulses of 5-min FUS stimulations caused a significant induction of gene expression as quantified by the Fluc/Rluc ratio of the cells assayed $8 \mathrm{hr}$ later (Fig. 3d, Methods).

144 The induction level is comparable to that of the positive control using thermal cycler with the same heating pattern (Fig. 3d), suggesting the acoustogenetic approach can remotely control gene activation in engineered cells with high efficiency.

We then used MRI-guided FUS to control local temperature in vivo in mouse (Fig. 3, e and f and Movie S2) and tested the FUS-induced gene activation using Nalm-6 cells with the dualluciferase reporter in vivo. Significant gene induction was observed in the implanted cells with only two pulses of 5-min FUS stimulation (FUS+, after), in comparison to the basal level (FUS+, before) and the control groups (FUS-, before and after) (Fig. 3, g and h).

\section{FUS-inducible tumor cytotoxicity of the engineered CAR T cells in vivo}

154 We next tested the tumor cytotoxicity of the FUS-inducible CAR T cells in vivo. We subcutaneously injected Nalm-6 cells (Fluc+) on both hindlimbs of NSG mice to generate matched bilateral tumors (Fig. 4a). Four days later, engineered CD19CAR T cells were

157 subcutaneously injected at both tumor sites locally, followed by three pulses of 5-min FUS 158 stimulation at $43^{\circ} \mathrm{C}$ on the left but not on the right tumor (Fig. 4a). Bioluminescence imaging 
161 local site had negligible off-site effects in attacking the distal tissues on the contralateral

162 hindlimb expressing the same antigens. We further performed a control experiment subjecting

163 mice carrying bilateral tumors to FUS stimulation on one side, with neither site subjected to the

164 injection of engineered CAR T cells (fig. S5a). The tumors with or without FUS stimulation

165 exhibited similar growth profiles, indicating that FUS itself (with the chosen pattern) had no

166 impact on tumor growth (fig. S5, b and c). Therefore, our results demonstrated that FUS can be

167 used to precisely control the cytotoxicity of the engineered CD19CAR T cells in vivo against

168 target tumor cells.

We further tested this acoustogenetic technology in controlling inducible CAR T cells against

171 other types of tumors, particularly solid tumors. We engineered solid tumor human prostate

172 cancer PC3 cells to express the prostate-specific membrane antigen (PSMA) and Fluc, and

173 engineered primary human T cells with the Cre-lox mediated heat-inducible anti-PSMA CAR

174 (PSMACAR; fig. S6a). We verified the functionality of the heat-inducible PSMACAR T cells

175 through in vitro co-culture cytotoxicity assays and the associated cytokine assays (fig. S6, b to

176 d). We then generated matched bilateral subcutaneous PC3 tumors (PSMA+, Fluc + ) in NSG

177 mice; five days later we subcutaneously injected heat-inducible PSMACAR T cells next to the

178 tumor sites on both sides. The tumor regions on the left side were treated with three pulses of 5-

179 min FUS, while those on the right remained unstimulated. Consistently, the tumors with FUS

180 stimulation showed significantly inhibited growth as compared to the controls (Fig. 4, d and e).

181 We further harvested the tumor tissues and quantified the related mRNA amount. The CD3

182 mRNA in the FUS-treated tumors averaged 3-fold of that in the untreated ones, indicating more

183 T cell infiltration in the FUS-treated solid prostate tumors (fig. S7a). Moreover, the amount of 
184 Cre-mediated recombined CAR mRNA in the FUS-treated tumors was 9-fold of that in the

185 untreated controls, verifying the FUS-induced DNA recombination and subsequent CAR

186 expression in the engineered T cells at the tumor sites (fig. S7, b and c, and Methods). These

187 results demonstrated the efficacy of FUS-based acoustogenetics in remote control of CAR T

188 cells for treating different types of tumors in vivo, including solid tumors of prostate cancer.

\section{Discussion}

We developed an FUS-based acoustogenetic approach to remotely control, without any exogenous cofactor, the genetically engineered $\mathrm{T}$ cells capable of perceiving ultrasound signals and transducing them into genetic and cellular activations for therapeutic applications in vivo. This acoustogenetics technology enables the activation of CAR T cells at confined tissue regions,

194 thus allowing the targeting of the less ideal antigens without causing non-specific off-site cytotoxicity. This is of critical importance given the extensive overlap of antigens between tumors and normal cells, particularly those under conditions of tissue injury and inflammation.

197 The short-pulsed patterns of FUS stimulation should also minimize potential detrimental effects

198 of hyperthermia and induce transient expression of synthetic protein regulators to circumvent severe immune responses. This acoustogenetic approach is highly modular, with the target CAR genes switchable to aim at different cancer types. 
as dihydrofolate reductase (DHFR) can be fused to CAR to control the protein lifetime with an FDA-approved drug methotrexate (22). This "AND” gate with FUS and methotrexate should enhance the precision of controllable CAR T immunotherapy.

211 We anticipate that comparable therapeutic outcomes can be achieved in a reversible heat-

212 inducible system without the Cre-lox gene switch, but this may require multiple rounds of FUS

213 stimulation. In such a system, Hsp directly drives the production of CAR (Hsp-CAR) under FUS

214 stimulation. Upon the withdrawal of FUS stimulation, HSFs gradually dissociate from Hsp,

215 returning Hsp and its downstream transcriptional activities to the resting state. This recovery

216 process is relatively fast, within 45 min after HS for Drosophila Hsp70 and approximately 60

217 min after HS for human $\operatorname{Hsp} 70(23,24)$. The dynamics of this heat-induced CAR expression

218 hence largely depends on its protein lifetime, with the half-life of GFP-tagged CAR reported to

219 be around $8 \mathrm{hr}(16)$. Therefore, repeated FUS stimulation can be applied to maintain the CAR

220 expression in the T cells (and hence their cytotoxicity) for a sustained period of time. We tested

221 this concept by applying a 10-min HS every $48 \mathrm{hr}$ in T cells with Hsp-eGFP, and indeed

222 observed oscillatory patterns of the induced eGFP expression (fig. S8). We anticipate that T cells

223 with a simple Hsp-CAR can also be repeatedly activated by FUS to achieve sustained CAR

224 expression and cytotoxicity for a desired period of time or until tumor elimination. Such a

225 reversible FUS-inducible system can further prevent "on-target off-tumor" toxicity of canonical

226 CAR T therapy, as the T cells leaving the tumor site will no longer receive FUS stimulation and

227 gradually lose CAR molecules. The tunable FUS pulses should also allow the precise control of

228 the temporal activation patterns of CAR T cells for an optimized killing efficiency with

229 controllable exhaustion. 
231 We chose local injection at the tumor site to deliver T cells in vivo. Local administration of CAR

232 T cells has been tested in animals and patients to overcome the obstacle of $\mathrm{T}$ cell homing

233 associated with intravenous delivery, and has achieved promising therapeutic effects $(16,25,26)$.

234 For example, since the prostate is positioned near critical organ structures including urethra and

235 neurovascular bundles, surgery or radiation therapy targeting the whole prostate gland to treat the

236 prevalent locally-progressed prostate cancer (27) may cause adverse effects that would

237 significantly impact quality of life $(28,29)$. Local delivery and activation of inducible CAR T

238 cells using clinically available MRI-guided FUS systems should allow, without any exogenously

239 added nanoparticle or cofactor, a high degree of precision and safety in eradicating tumor cells in

240 these patients harboring locally progressed prostate cancer (29). In cases where intravenous

241 delivery is required, it is also possible to equip the FUS-inducible CAR T cells with additional

242 antigen binders and/or chemokine receptors to promote trafficking, infiltration, and the

243 enrichment of these engineered cells at the tumor site before FUS activation $(30,31)$.

245 The short-pulsed stimulation and the biocompatible Hsp capable of inducing transient

246 expressions of different synthetic protein regulators can potentially enhance the safety of gene

247 therapy, circumventing detrimental host immune response. For instance, CRISPR-Cas9 proteins

248 have been a powerful tool for research in genetic and epigenetic engineering, but can evoke

249 adaptive immune responses and tissue damage in vivo, and are therefore potentially pathogenic if

250 applied to correct inherited genetic defects to treat diseases (32). Protein engineering to remove

251 immunogenic epitopes and humanize these synthetic proteins to circumvent this issue can be

252 difficult owing to the high diversity of the human leukocyte antigen (HLA) loci (33). Using our 
acoustogenetic approach, the transiently induced Hsp-driven synthetic regulators (e.g. Cas9) can

254 be cleared in a timely manner to mitigate or evade the adaptive immune response, hence offering a new option for gene therapy.

257 Each component of this FUS-based acoustogenetics, i.e. ultrasound devices, molecular thermo-

258 sensors, and genetic/epigenetic transducing modules, is highly modular and will continue to evolve

259 for greater precision and reduced immunogenicity. In fact, stretchable electronic circuits are being

260 developed to fabricate wearable patches of ultrasound transducers (34). The leverage of

261 technological advancements of different fields into FUS-based acoustogenetics should be able to

262 drive the development of these fields to open up new frontiers. We envision that the current state

263 of acoustogenetics is analogous to optogenetics at its infancy. Before the functional demonstration

264 of channelrhodopsin in neuronal cells (35), it was challenging to manipulate molecular activities in

265 live cells at high spatiotemporal resolutions. With the technological integration and convergence of

266 optics, genetic circuits, and light-sensitive proteins, optogenetics is rapidly reaching its full potential.

267 Based on this analogy, acoustogenetics may undergo a similar trajectory to provide a broadly

268 applicable method and usher in an era of applying ultrasound for the direct, remote, and noninvasive

269 control of genetically engineered cells for therapeutics.

\section{Methods}

\section{Cloning}

272 Plasmids used in this paper are listed in Table S1. Cloning strategies include Gibson Assembly

273 (NEB, E2611L) and T4 ligation (NEB, M0202L). PCR was performed using synthesized primers

274 (Integrated DNA Technologies) and Q5 DNA polymerase (NEB, M0491). The sequences of the 275 constructed plasmids were verified by Sanger sequencing (Genewiz). 
General cell culture

HEK 293 T cells were cultured in DEME (Gibco, 11995115) with 10\% FBS (Gibco, 10438026) and 1\% Penicillin-Streptomycin (Gibco, 15140122). Jurkat, Nalm-6, and PC3 cells were cultured in RPMI 1640 (Gibco, 22400105) with 10\% FBS and 1\% P/S. Primary human T cells were cultured in complete RPMI 1640 supplemented with $100 \mathrm{U} / \mathrm{mL}$ recombinant human IL-2 (PeproTech, 200-02). Cells were cultured at $37^{\circ} \mathrm{C}$ in a humidified $5 \% \mathrm{CO}_{2}$ incubator.

\section{Staining and flow cytometry}

285 Staining of cell surface markers (e.g., c-Myc, CD69, etc) for flow cytometry was performed 286 using fluorophore-conjugated antibodies according to manufacturers' protocols. In general, cells 287 were washed twice and resuspended in $100 \mu \mathrm{L}$ wash buffer (PBS $+0.5 \%$ BSA) containing the 288 suggested amounts of antibodies, incubated in dark at room temperature for suggested durations, and washed three times before being analyzed using a BD flow cytometer. Gating was based on non-engineered cells with the same staining. Flow cytometry data were analyzed using FlowJo 291 software (Tree Star).

\section{In vitro heat shock}

294 For Fig. 1c and Movie S1, cells seeded in a glass bottom dish were heated at $43^{\circ} \mathrm{C}$ for $15 \mathrm{~min}$ 295 using a heating stage (Instec) integrated with a Nikon Eclipse Ti inverted microscope. Images 296 were acquired in real-time to obtain the kinetics of the induced fluorescent protein. For the 297 remainder of the in vitro heat shock (HS) experiment, unless otherwise specified, cells were 298 washed and resuspended in cell culture medium at a concentration of $2 \times 10^{6}$ cells $/ \mathrm{mL}$, aliquoted 
into 8-strip PCR tubes with $50 \mu \mathrm{L} /$ tube, and heat shocked at $43^{\circ} \mathrm{C}$ using a thermal cycler (BioRad, 1851148) with various patterns as indicated (Table S2). Cells were returned to standard culture condition after HS. The gene induction levels were quantified by flow cytometry $12 \mathrm{hr}$

302 after HS in Fig. 1, f and g, and fig. S3, d and e.

\section{Engineered cells}

305 The engineered cells (excluding primary human T cells) used in this work are listed in Table S3. Lentiviruses were used to deliver engineered genes into the cells. Fluorescence-activated cell sorting (FACS), when needed, was performed at UCSD Human Embryonic Stem Cell Core Facility by professional technicians following standard protocols.

\section{Quantification of CAR expression in Jurkat cells}

311 Jurkat cells were either transduced with a lentiviral cocktail (inducible Cre and lox-stop CAR 312 reporter, Fig. 2a) followed by indicated HS (Fig. 2b), or transduced with the lox-stop CAR

313 reporter lentivirus alone without HS. CAR expression was quantified by CAR antibody staining

314 (an anti-mouse IgG, F(ab') ${ }_{2}$ fragment specific antibody; Jackson ImmunoResearch, 115-606-

315 072) and flow cytometry $24 \mathrm{hr}$ after HS. Live single cells were gated for CAR expression

316 analysis. Non-engineered Jurkat cells were stained with the same antibody to generate the CAR+ 317 gate.

\section{Quantification of CD69 expression in Jurkat cells}

320 Jurkat cells transduced with a lentiviral cocktail (inducible Cre and lox-stop CAR reporter, Fig.

$3212 \mathrm{a})$ were treated with or without $\mathrm{HS}$ at $43^{\circ} \mathrm{C}$ for $15 \mathrm{~min}$, and co-cultured with target tumor cells 
322 for $24 \mathrm{hr}$. The cells were then stained by an APC anti-human CD69 antibody (BioLegend,

323 310910) and analyzed by flow cytometry. ZsGreen+ cells (representing the engineered Jurkat

324 cells) were gated for analysis of CD69 expression. Non-engineered Jurkat cells co-cultured with

325 target tumor cells were stained with the same antibody to generate the CD69+ (APC+) gate.

327 Isolation, culture, transduction and MACS of primary human T cells

328 Human peripheral blood mononuclear cells (PBMCs) were isolated from buffy coats (San Diego

329 Blood Bank) using Lymphocyte Separation Medium (Corning, 25-072-CV) following the

330 manufacturer's instructions. Primary human T cells were isolated from PBMCs using Pan T Cell

331 Isolation Kit (Miltenyi, 130-096-535) and activated with Dynabeads® Human T-Expander

332 CD3/CD28 (Gibco, 11141D). Three days later, lentivirus concentrated using PEG-it (SBI,

333 LV825A-1) was added to the T cells at MOI = 10, followed by spinoculation in a 96-well plate

334 coated with Retronectin (Takara, T100B). T cells were further expanded and dynabeads were

335 removed prior to downstream procedures (e.g., magnetic-activated cell sorting (MACS), in vitro

336 HS, in vivo injection, etc.).

338 For in vitro and in vivo cytotoxicity studies, T cells were transduced with a lentiviral cocktail of

339 inducible Cre and lox-stop CAR reporter (Fig. 2a). MACS was performed using Anti-c-Myc-

340 Biotin antibodies and Anti-Biotin microbeads (Miltenyi, 130-092-471 and 130-097-046)

341 following the manufacturer's instructions to enrich c-Myc+ cells. A representative double

342 positive efficiency after MACS is $69 \%, 95 \%$ for the c-Myc+ and $71.4 \%$ for the ZsGreen+ cells

343 (fig. S2b). CAR expression in the engineered inducible CAR T cells with or without HS was

344 quantified using the CAR antibody as described above. 


\section{Luciferase-based cytotoxicity assay}

347 A constant number of $5 \times 10^{4}$ Fluc + Nalm- 6 cells were mixed with engineered primary human $\mathrm{T}$

348 cells with or without HS (pre-washed and resuspended with complete RPMI without IL-2) at

349 effector-to-target (E:T) ratios of 1:50, 1:20, 1:10, 1:5, 1:1, 5:1 or no T cells (“target cell only").

350 The mixtures were then cultured in round bottom 96 well plates for $24 \mathrm{hr}$, centrifuged to remove

351 the supernatant (which was harvested for quantification of cytokine production), and assayed

352 with the Bright-Glo ${ }^{\mathrm{TM}}$ Luciferase Assay System (Promega, E2610) following the manufacturer's

353 instructions to quantify the luminescence of each sample. The cytotoxicity (\%) of Sample X was

354 calculated as (1- Luminescence of X / Luminescence of “target cell only") x 100\%.

356 For cytotoxicity assay using PC3 cells as the target, $1 \times 10^{4}$ PSMA + Fluc + PC3 cells were

357 seeded onto TC-treated flat bottom 96 well plates (Corning, 3603). Except for "target cell only"

358 wells, engineered primary human T cells with or without HS (washed and resuspended with

359 complete RPMI without IL-2) were added $6 \mathrm{hr}$ later at E:T ratios of 1:10, 1:5, 1:1, 5:1, 10:1,

360 20:1. The luminescence was quantified $24 \mathrm{hr}$ after co-culture as described above.

\section{Quantification of cytokine production}

363 The supernatant of effector-target cell co-culture was harvested. The concentrations of cytokines

364 IL-2 and IFN- $\gamma$ were quantified using the corresponding ELISA kits (BD, 555190 and 555142). 
367 Non-engineered primary human T cells were heat shocked as described above and kept under

368 normal culture condition for $24 \mathrm{hr}$. Cell viability was then assessed using the FITC Annexin V

369 Apoptosis Detection Kit I (BD, 556547) following the manufacturer's instructions. The cells

370 stained negative for both Annexin V and PI were counted as live cells.

\section{$372 \quad$ MRI-guided FUS}

373 The MRI-guided FUS system is composed of a $1.5 \mathrm{MHz} 8$-element annular array transducer, a

374 16-channel broadband RF generator, a piezo motor-based X-Y positioning stage, and a degassing

375 and water circulation system (Image Guided Therapy, France). MR images acquired using a

376 Bruker 7T MRI system were transferred to ThermoGuide software (Image Guided Therapy,

377 France) to generate phase images and real-time temperature maps. Using PID controller, the

378 software automatically regulates the output power of the generator to maintain the temperature at

379 the focal spot at a desired value as described elsewhere $(6,36)$.

Animal experiments were performed following Protocol S15285 approved by UCSD IACUC.

382 NSG mice (6-8 weeks old) were purchased from Jackson Laboratory (JAX) and shaved prior to

383 FUS stimulation. Anesthesia was induced using 2\% isoflurane-oxygen mixture and maintained

384 with $1.5 \%$ isoflurane-oxygen mixture during FUS stimulation. The mouse was laid on its side on

385 an MR bed containing an agarose gel pad and a surface coil. A pressure pad was placed under

386 the mouse to monitor its respiration rate, and a rectal thermal probe was used to provide

387 feedback for the delivering of warm air into the bore to maintain the mouse's core temperature at

388 approximately $37^{\circ} \mathrm{C}$. The ultrasound transducer was positioned right above the targeted region 
on the mouse's hindlimb. Thin layers of SCAN ultrasound gel (Parker labs) were applied at the skin-transducer and skin-bed interfaces.

392 The ThermoGuide software regulates the temperature in a $3 \times 3$ pixel square $\left(3-4 \mathrm{~mm}^{2}\right)$

393 centered at the ultrasound focus (Fig. 3e). A PID controller is used to maintain the average

394 temperature of the target square at $6^{\circ} \mathrm{C}$ above reference by controlling the output power of the

395 FUS generator, with the reference temperature being $37^{\circ} \mathrm{C}$ as measured by the rectal thermal

396 probe. As such, the MRI-guided FUS enabled temperature elevation to $43^{\circ} \mathrm{C}$ locally at the focal

397 area in the hindlimb of an anesthetized mouse.

\section{FUS stimulation in tofu phantom}

400 For FUS stimulation on cells in the tofu phantom, Nalm-6 cells were lentivirally transduced with 401 the dual-luciferase reporter (Fig. 3b, Hsp-Fluc-PGK-Rluc-mCherry) and FACS-sorted. The cells 402 were resuspended in culture medium and mixed with matrigel (Corning, 354262) at 1:1 volume 403 ratio on ice. Extra-firm tofu was cut into a 15-mm thick pad, and an 8-mm deep hole of 8-mm 404 diameter was drilled from the top. A microcentrifuge tube of 7.5-mm diameter (Fisherbrand, 05405 408-120) was cut to 8-mm long by removing the lid and the conical bottom, and was inserted 406 into the hole in the tofu phantom. Cell-matrigel mixture of $150 \mu \mathrm{L}$ was added into the hole $(\sim 3$ $407 \mathrm{~mm}$ thick) and allowed to gel at room temperature. The rest of the hole and the gap between the 408 tube and the tofu phantom were filled up with matrigel. After gelation, the assembly was 409 inverted and positioned onto the MR bed containing the surface coil. The ultrasound transducer 410 was positioned above the tofu phantom with its center aligned with that of the tube. Thin layers 
411 of ultrasound gel were applied at the tofu-transducer and tofu-bed interfaces. A thermal probe

412 was inserted into the distal end of the tofu phantom to provide reference temperature readings.

414 MR images of the assembly were acquired and transferred to ThermoGuide to calculate the

415 theoretical ultrasound focal position. Test FUS shots were delivered to determine the actual focal

416 position. Steering was applied to focus the ultrasound at the region immediately above the cells.

417 Three pulses of 5-min FUS stimulations at $43^{\circ} \mathrm{C}$ were applied. The cell-matrigel mixture was

418 then recovered from the tube, placed in cell culture medium, and returned to a standard $37^{\circ} \mathrm{C}$ cell

419 culture incubator. After $6 \mathrm{hr}$, the culture was centrifuged to remove the supernatant, and the cell-

420 matrigel pellet was incubated in a Cell Recovery Solution (Corning, 354253) at $4^{\circ} \mathrm{C}$ for $1 \mathrm{hr}$ to

421 retrieve the Nalm-6 cells from matrigel. The Fluc and Rluc luminescence of the cells was

422 quantified using the Dual-Luciferase ${ }^{\circledR}$ Reporter Assay System (Promega, E1910) following the

423 manufacturer's instructions.

\section{In vivo bioluminescence imaging}

426 In vivo bioluminescence imaging (BLI) was performed using an In vivo Imaging System (IVIS)

427 Lumina LT Series III (PerkinElmer). For Fluc imaging, 150 mg/kg D-Luciferin (GoldBio,

428 LUCK) was administered intraperitoneally (i.p.). BLI started 10 min after substrate injection

429 until peak signal was acquired. For Rluc imaging, $200 \mu \mathrm{L} 0.295$ mM ViviRen ${ }^{\mathrm{TM}}$ (Promega,

430 P1232) (37) was administered i.p. BLI started 15 min after substrate injection until peak signal

431 was acquired. BLI of Fluc and Rluc in the same mouse, when needed, was performed $4 \mathrm{hr}$ apart.

432 Images were analyzed using Living Image software (PerkinElmer), and the integrated Fluc

433 luminescence intensities within regions of interest were quantified to represent tumor sizes. 
FUS-inducible gene activation in vivo

NSG mice (male, 6-8 weeks old) were subcutaneously injected with 2 × $10^{6}$ dual-luciferase reporter Nalm-6 cells at the hindlimb. One week later, the experimental mice received two pulses of 5-min FUS stimulation at $43^{\circ} \mathrm{C}$ targeted at the implanted cells, while the control mice remained unstimulated. The in vivo Fluc and Rluc luminescence was quantified $4 \mathrm{hr}$ before and $12 \mathrm{hr}$ after FUS stimulation, as described above.

\section{In vivo tumor cytotoxicity of FUS-inducible CAR T cells.}

443 NSG mice (male, 6-8 weeks old) were subcutaneously injected with 2 x $10^{5}$ Fluc + Nalm-6 cells

444 (or $2 \times 10^{5}$ PSMA+ Fluc+ PC3 cells, for PC3 tumors) on both hindlimbs to generate matched

445 bilateral tumors. Four days later (or five days later, for PC3 tumors), 1 x $10^{6}$ inducible primary

446 human CAR T cells prepared as described above were injected subcutaneously and locally at

447 tumor regions. Within $4-8 \mathrm{hr}$ after T cell injection, three pulses of 5-min FUS stimulation

448 targeted at $43^{\circ} \mathrm{C}$ were applied on the left tumor region as described above, while the tumor on the

449 right hindlimb received no FUS stimulation to serve as the control. Tumor aggressiveness was

450 monitored by BLI twice a week as described above until euthanasia criteria were met.

Quantification of mRNA expression in tumor tissue.

453 PC3 tumors (Fig. 4, d and e) were harvested 22 days after tumor implantation (17 days after T

454 cell injection and FUS stimulation). The tumors were disrupted and homogenized, and the same

455 amount of lysate from each tumor was used to extract total RNA with the RNeasy Mini Kit

456 (Qiagen, 74104) followed by reverse transcription using the same amount of template RNA. 


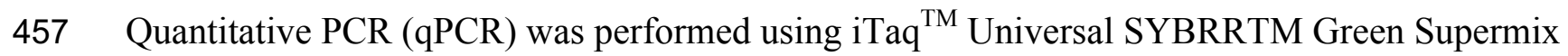

458 (Bio-Rad, 1725121), the same amount of template cDNA, and the specific primers described

459 below. The mRNA levels were normalized to of-actin.

461 The first pair of specific primers were designed on human CD3 $\gamma$ chain to detect the presence of

462 human T cells. The second pair of specific primers were designed based on the lox-stop

463 PSMACAR reporter sequence to reflect CAR expression after FUS-induced Cre recombination

464 (fig. S6, a and b). The forward primer anneals from -60 bp of the mouse PGK promoter,

465 downstream of the transcription starting site (TSS), and the reverse primer anneals from $+20 \mathrm{bp}$

466 of the PSMACAR gene (38). With the presence of FUS-induced Cre recombinase, the sequence

467 from the second half of LoxH to the first half of LoxP will be excised, resulting in a $200 \mathrm{bp}$

468 qPCR product. Without Cre-mediated recombination, this pair of primers will theoretically

469 generate a 984-bp fragment. We adopted a two-step qPCR protocol with combined

470 annealing/extension at $60^{\circ} \mathrm{C}$ for only $15 \mathrm{sec}$ to ensure the specific amplification of the $200-\mathrm{bp}$

471 fragment, but not the $984 \mathrm{bp}$ fragment, as confirmed by gel electrophoresis and Sanger

472 sequencing of the qPCR product (fig. S6, c and d; sequence alignment performed in Serial

473 Cloner). Therefore, the second pair of specific primers can detect the successfully recombined

474 CAR mRNA amount.

475

$476 \quad$ Statistics.

477 One-way ANOVA followed by Tukey's multiple comparisons test is used for Figs. 1, f and g, and

478 fig. S3, b and d to e. Student's t-test is used for Fig. 2d. Two-way ANOVA followed by Sidak's

479 multiple comparisons test is used for Figs. 2, b, g to I, 3g, 4, b and d, fig. S4b, S5, b to d. 


\section{Acknowledgments}

481 We thank Dr. Franck Couillaud (University of Bordeaux, France) for providing the Hsp template

482 and Dr. Michel Sadelain (Sloan Kettering Institute, USA) for the PSMA scFv and PSMA

483 constructs and the Nalm-6 cells. We also thank Dr. Erik Dumont and Ms. Stéphanie Hoarau-

484 Recco (Image Guided Therapy, France) for their most valuable help on the FUS system.

485 Funding: This work was supported in part by grants from NIH HL121365, GM125379,

486 GM126016, CA204704 and CA209629 (Y. Wang). Author contributions: Y.Wu, S.C. and

487 Y.Wang designed research; Y.Wu, Y.L, Z.H., X.W., Z.J., J.L., P.L., L.Z., M.A., Y.P., R.B., A.J.

488 performed research; Y.Wu and Y.L. analyzed data; Y.Wu, T. L., S.C. and Y.Wang wrote the

489 manuscript. All authors reviewed the manuscript and have given approval to the final version of

490 the manuscript. Competing interests: Y.Wang is a scientific co-founder of Cell E\&G Inc and

491 Acoustic Cell Therapy Inc. These financial interests do not affect the design, conduct or

492 reporting of this research. Data and materials availability: All data is available in the main text

493 or the supplementary materials.

\section{Figure legends}

495 Fig. 1. Heat-inducible gene activation. (a) Design of the FUS-controllable CAR T therapy

496 technology. T cells engineered with the heat-inducible CAR and localized at the tumor region are

497 activated by MRI-guided FUS for recognizing and eradicating target tumor cells. (b) Schematics

498 of the dual-promoter eGFP reporter. (c-d) (c) Fluorescent images of inducible eGFP and

499 constitutive mCherry, and (d) the percentage of eGFP+ cells and their mean fluorescence

500 intensity after a $15-\mathrm{min} \mathrm{HS}$ at $43^{\circ} \mathrm{C}$ in $\mathrm{HEK} 293 \mathrm{~T}$ cells containing the dual-promoter reporter. (e-

501 g) Gene induction in primary human T cells with the dual-promoter eGFP reporter. (e) 
Representative flow cytometry profiles of eGFP expression. (f) The percentage of eGFP+ cells and (g) their mean fluorescence intensity. In (e-g), CT: without HS; HS: with a continuous 15min HS. MCherry+ cells were gated for eGFP analysis. $\mathrm{N}=3$ repeats; error bar: SEM. ****: $\mathrm{p}<$

5050.0001.

\section{Fig. 2. Heat-inducible CD19CAR expression and functional outcomes in Jurkat and} reporter. (b) Inducible CAR expression in Jurkat cells hosting the lox-stop CAR reporter alone (lox), or both transgenes in (a) with (HS) or without HS (CT). (c) Schematics of assays accessing the functionality of the heat-induced CAR T cells, including CD69 expression, cytotoxicity, and cytokine release. (d) The percentage of CD69+ cells in Jurkat with both transgenes in (a). (e) Representative flow cytometry data showing the histogram of CD69 expression in (d). (f)

514 Representative histograms showing the percentage of CAR + cells in primary $\mathrm{T}$ cells with both transgenes in (a). (g) The cytotoxicity of the T cells in (f) against Nalm-6 tumor cells at various E:T ratios. (h-i) Quantification of (h) IFN- $\gamma$ and (i) IL-2 cytokine release associated with (g).

517 Arrow: cytokine level not detectable. In (b) and (d) to (f), CT: without HS; HS: with a

518 continuous 15-min HS. $\mathrm{N}=3$; error bar: SEM. ***: $\mathrm{p}<0.001 ; * * * *: \mathrm{p}<0.0001$; ns: no 519 significant difference.

521 Fig. 3. MRI-guided FUS-inducible gene activation in phantom and in vivo. (a) Schematics of 522 the MRI-guided FUS system. (b) The dual-luciferase reporter containing the inducible Hsp523 driven Fluc and constitutive PGK-driven Rluc fused with mCherry. (c) The experimental setup 524 of FUS stimulation on cells in a tofu phantom. (d) Gene induction level in Nalm-6 cells 
containing the dual-luciferase reporter with three pulses of 5-min heating by MRI-guided FUS in

526

527

528

529

530

531

532

533

534

535

536

537

538

539

540

541

542

543

544

545

546

547

tofu phantom (FUS) or by thermal cycler (HS). CT: without heating. Gene induction level is quantified by the Fluc/Rluc ratio and normalized to $\mathrm{CT} . \mathrm{N}=3$. (e) Left: color-coded temperature map superimposed on MRI images at different time points during a 5-min FUS stimulation at $43^{\circ} \mathrm{C}$ on the hindlimb of an anesthetized mouse. Right: close-up of the red rectangle region on the left. The dotted white square outlines the region of interest (ROI) for temperature regulation. (f) The average temperature of the ROI during FUS stimulation in (e). The yellow shadow represents the predefined target temperature $\left(43^{\circ} \mathrm{C}\right)$ and duration $(300 \mathrm{sec})$ of FUS stimulation. (g) Gene induction in vivo by MRI-guided FUS. Nalm-6 cells containing the dual-luciferase reporter were injected subcutaneously into NSG mice followed by FUS stimulation. FUS+ or FUS-: with or without two pulses of 5-min FUS stimulation at $43^{\circ} \mathrm{C}$. Gene induction was quantified by the in vivo Fluc/Rluc ratio and normalized to the "FUS-, before" group, as indicated by the dotted line. $\mathrm{N}=4$ mice. (h) Representative bioluminescence images of Fluc expression before and after FUS stimulation in (g). Error bar: SEM. *: $\mathrm{p}<0.05 ; * *$ : $\mathrm{p}<0.01$; ns: no significant difference.

\section{Fig. 4. FUS-controllable tumor suppression by the engineered CAR T cells in vivo. (a)}

Timeline of the in vivo experiment using NSG mouse bearing matched bilateral tumors as the animal model. The tumor on the left flank received FUS stimulation (FUS+) and the one on the right received no FUS (FUS-) following injection of engineered CAR T cells. (b-e) The quantified tumor growth and representative bioluminescence images of (b-c) Nalm-6 tumors or (d-e) PC3 tumors with (FUS+) or without (FUS-) FUS stimulation. Tumor size was quantified using the integrated Fluc luminescence intensity of the tumor region and normalized to that of 
the same tumor on the first measurement. $\mathrm{N}=4$ mice. Error bar: SEM. ${ }^{*}: \mathrm{p}<0.05 ; * *: \mathrm{p}<0.01$;

$* * * *: \mathrm{p}<0.0001$

\section{References}

1. R. Y. Tsien, Imagining imaging's future. Nat Rev Mol Cell Biol Suppl, SS16-21 (2003).

2. M. Thanou, W. Gedroyc, MRI-Guided Focused Ultrasound as a New Method of Drug Delivery. J Drug Deliv 2013, 616197 (2013).

3. R. Deckers et al., Image-guided, noninvasive, spatiotemporal control of gene expression. Proceedings of the National Academy of Sciences of the United States of America 106, 1175-1180 (2009).

4. E. Guilhon et al., Image-guided control of transgene expression based on local hyperthermia. Mol Imaging 2, 11-17 (2003).

5. S. Wang, V. Zderic, V. Frenkel, Extracorporeal, low-energy focused ultrasound for noninvasive and nondestructive targeted hyperthermia. Future Oncol 6, 1497-1511 (2010).

6. D. I. Piraner, M. H. Abedi, B. A. Moser, A. Lee-Gosselin, M. G. Shapiro, Tunable thermal bioswitches for in vivo control of microbial therapeutics. Nature chemical biology 13, $75-$ 80 (2017).

7. M. L. Davila et al., Efficacy and toxicity management of 19-28z CAR T cell therapy in B cell acute lymphoblastic leukemia. Science translational medicine 6, 224ra225 (2014).

8. D. Chakravarti, W. W. Wong, Synthetic biology in cell-based cancer immunotherapy. Trends in biotechnology 33, 449-461 (2015).

9. M. V. Maus, S. A. Grupp, D. L. Porter, C. H. June, Antibody-modified T cells: CARs take the front seat for hematologic malignancies. Blood 123, 2625-2635 (2014).

10. R. A. Morgan et al., Case report of a serious adverse event following the administration of $\mathrm{T}$ cells transduced with a chimeric antigen receptor recognizing ERBB2. Molecular therapy : the journal of the American Society of Gene Therapy 18, 843-851 (2010).

11. G. Akpek, S. M. Lee, V. Anders, G. B. Vogelsang, A high-dose pulse steroid regimen for controlling active chronic graft-versus-host disease. Biol Blood Marrow Transplant 7 , 495-502 (2001).

12. A. Di Stasi et al., Inducible apoptosis as a safety switch for adoptive cell therapy. The New England journal of medicine 365, 1673-1683 (2011).

13. M. Themeli, M. Sadelain, Combinatorial Antigen Targeting: Ideal T-Cell Sensing and Anti-Tumor Response. Trends Mol Med 22, 271-273 (2016).

14. J. H. Cho, J. J. Collins, W. W. Wong, Universal Chimeric Antigen Receptors for Multiplexed and Logical Control of T Cell Responses. Cell 173, 1426-1438 e1411 (2018).

15. V. D. Fedorov, M. Themeli, M. Sadelain, PD-1- and CTLA-4-based inhibitory chimeric antigen receptors (iCARs) divert off-target immunotherapy responses. Science translational medicine 5, 215ra172 (2013).

16. K. T. Roybal et al., Precision Tumor Recognition by T Cells With Combinatorial AntigenSensing Circuits. Cell 164, 770-779 (2016). 
590

591

592

593

594

595

596

597

598

599

600

601

602

603

604

605

606

607

608

609

610

611

612

613

614

615

616

617

618

619

620

621

622

623

624

625

626

627

628

629

630

631

632

633

634

635

636

637

638

639

640

17. C. Y. Wu, K. T. Roybal, E. M. Puchner, J. Onuffer, W. A. Lim, Remote control of therapeutic T cells through a small molecule-gated chimeric receptor. Science $\mathbf{3 5 0}$, aab4077 (2015).

18. M. M. D'Aloia, I. G. Zizzari, B. Sacchetti, L. Pierelli, M. Alimandi, CAR-T cells: the long and winding road to solid tumors. Cell Death Dis 9, 282 (2018).

19. S. I. Grivennikov, F. R. Greten, M. Karin, Immunity, inflammation, and cancer. Cell 140, 883-899 (2010).

20. Y. Pan et al., Mechanogenetics for the remote and noninvasive control of cancer immunotherapy. Proceedings of the National Academy of Sciences of the United States of America 115, 992-997 (2018).

21. I. C. Miller, M. Gamboa Castro, J. Maenza, J. P. Weis, G. A. Kwong, Remote Control of Mammalian Cells with Heat-Triggered Gene Switches and Photothermal Pulse Trains. ACS Synth Biol 7, 1167-1173 (2018).

22. M. V. Raimondi et al., DHFR Inhibitors: Reading the Past for Discovering Novel Anticancer Agents. Molecules 24, (2019).

23. K. Abravaya, B. Phillips, R. I. Morimoto, Attenuation of the heat shock response in HeLa cells is mediated by the release of bound heat shock transcription factor and is modulated by changes in growth and in heat shock temperatures. Genes Dev 5, 21172127 (1991).

24. S. K. Ghosh, A. Missra, D. S. Gilmour, Negative elongation factor accelerates the rate at which heat shock genes are shut off by facilitating dissociation of heat shock factor. Mol Cell Biol 31, 4232-4243 (2011).

25. M. Martinez, E. K. Moon, CAR T Cells for Solid Tumors: New Strategies for Finding, Infiltrating, and Surviving in the Tumor Microenvironment. Front Immunol 10, 128 (2019).

26. P. Sridhar, F. Petrocca, Regional Delivery of Chimeric Antigen Receptor (CAR) T-Cells for Cancer Therapy. Cancers (Basel) 9, (2017).

27. U. Mahmood et al., Current clinical presentation and treatment of localized prostate cancer in the United States. J Urol 192, 1650-1656 (2014).

28. H. B. Musunuru et al., Active Surveillance for Intermediate Risk Prostate Cancer: Survival Outcomes in the Sunnybrook Experience. J Urol 196, 1651-1658 (2016).

29. A. R. Rastinehad et al., Gold nanoshell-localized photothermal ablation of prostate tumors in a clinical pilot device study. Proc Natl Acad Sci U S A 116, 18590-18596 (2019).

30. M. Boice et al., Loss of the HVEM Tumor Suppressor in Lymphoma and Restoration by Modified CAR-T Cells. Cell 167, 405-418 e413 (2016).

31. K. T. Roybal et al., Engineering T Cells with Customized Therapeutic Response Programs Using Synthetic Notch Receptors. Cell 167, 419-432 e416 (2016).

32. W. L. Chew et al., A multifunctional AAV-CRISPR-Cas9 and its host response. Nat Methods 13, 868-874 (2016).

33. A. M. Moreno et al., Immune-orthogonal orthologues of AAV capsids and of Cas9 circumvent the immune response to the administration of gene therapy. Nat Biomed Eng 3, 806-816 (2019).

34. C. H. Wang et al., Monitoring of the central blood pressure waveform via a conformal ultrasonic device. Nat Biomed Eng 2, 687-695 (2018).

35. E. S. Boyden, F. Zhang, E. Bamberg, G. Nagel, K. Deisseroth, Millisecond-timescale, genetically targeted optical control of neural activity. Nature neuroscience 8, 1263-1268 (2005).

36. B. Z. Fite et al., Magnetic resonance thermometry at 7T for real-time monitoring and correction of ultrasound induced mild hyperthermia. PloS one 7, e35509 (2012).

37. M. Otto-Duessel et al., In vivo testing of Renilla luciferase substrate analogs in an orthotopic murine model of human glioblastoma. Mol Imaging 5, 57-64 (2006). 
bioRxiv preprint doi: https://doi.org/10.1101/2020.02 18.955005; this version posted February 19, 2020. The copyright holder for this preprint

(which was not certified by peer review) is the author/funder, who has granted bioRxiv a license to display the preprint in perpetuity. It is made available under aCC-BY-NC-ND 4.0 International license.

641 38. M. W. McBurney et al., The mouse Pgk-1 gene promoter contains an upstream activator

642 sequence. Nucleic Acids Res 19, 5755-5761 (1991).

643 


\section{Figure 1}

a

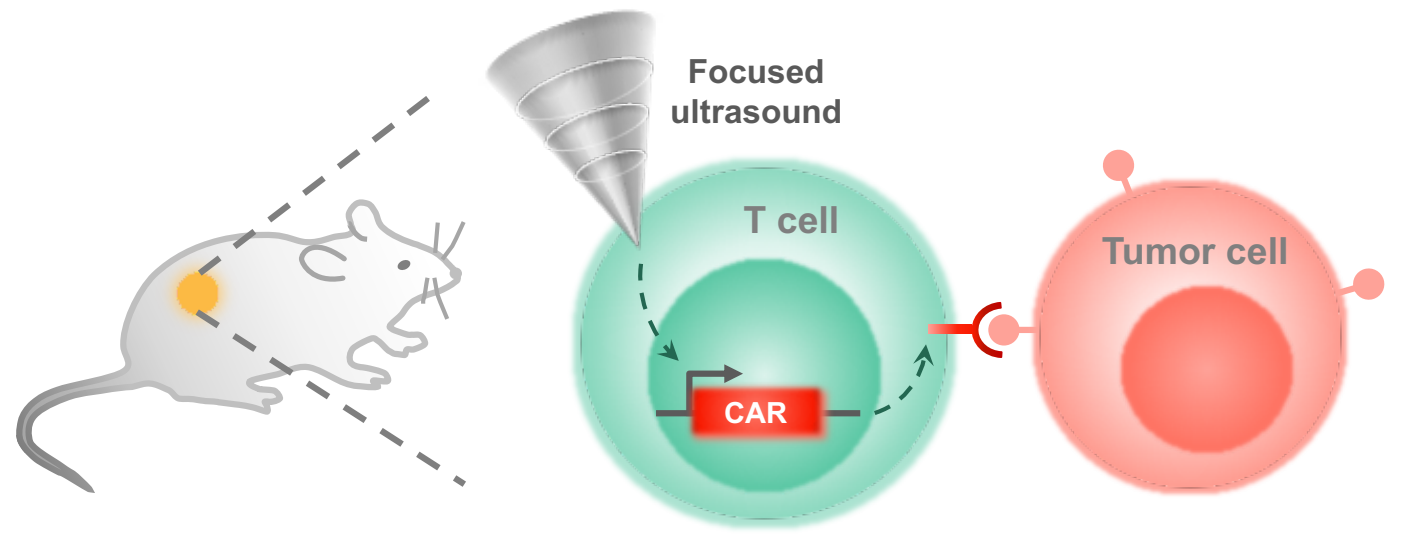

b

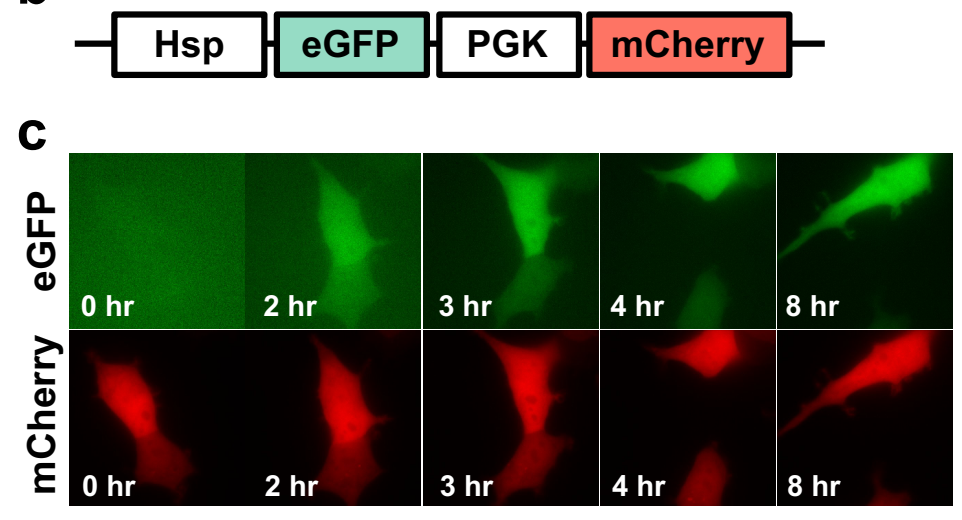

e

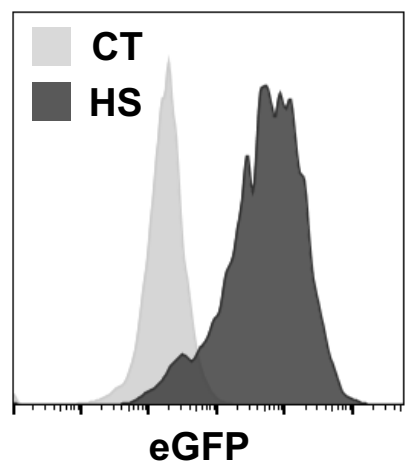

d O- eGFP+cells (\%)

$\triangle$ Mean intensity (AU)

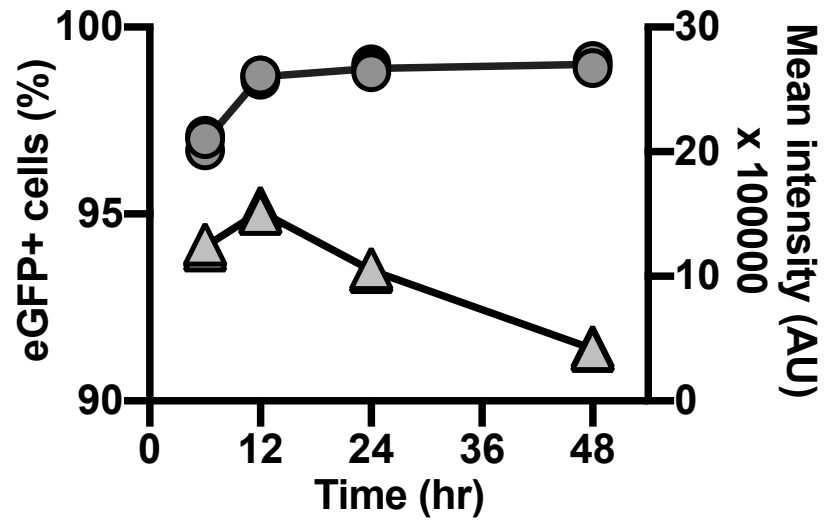

g

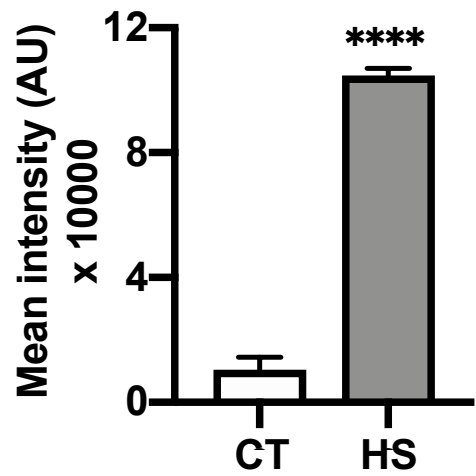




\section{Figure 2}

a

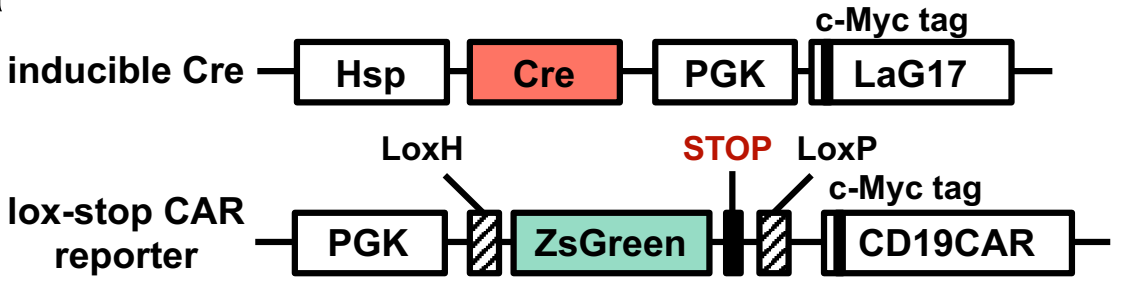

b

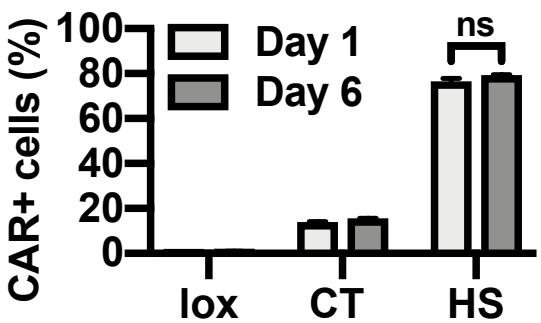

C

d

e
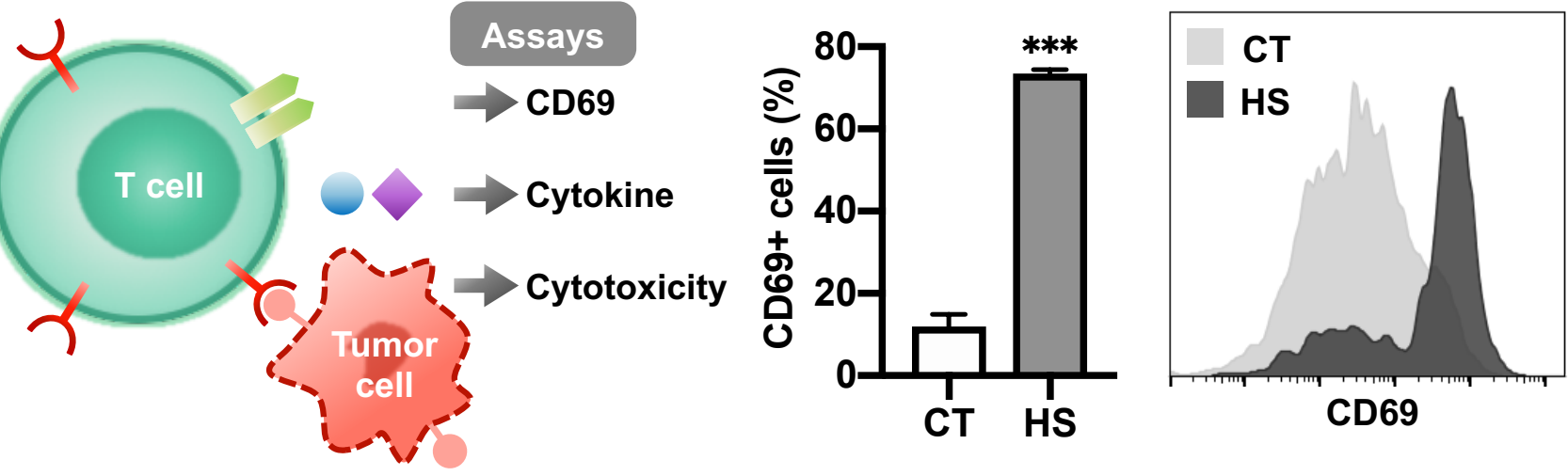

f

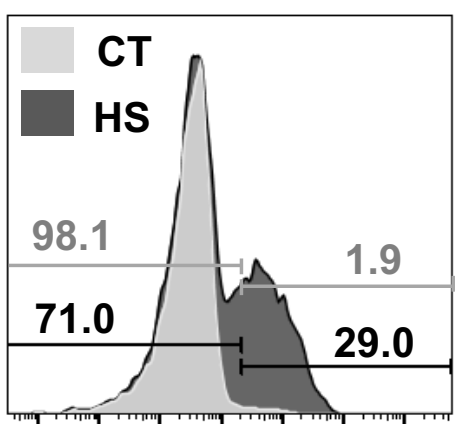

CD19CAR

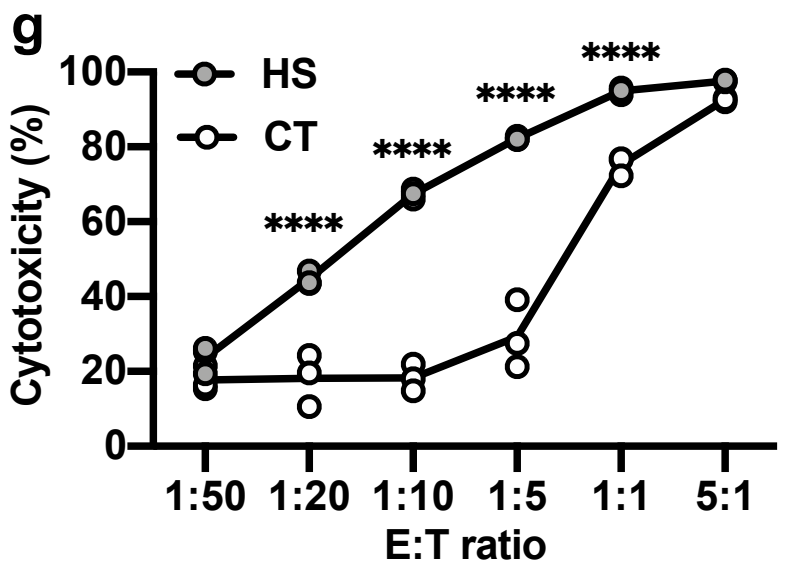

h
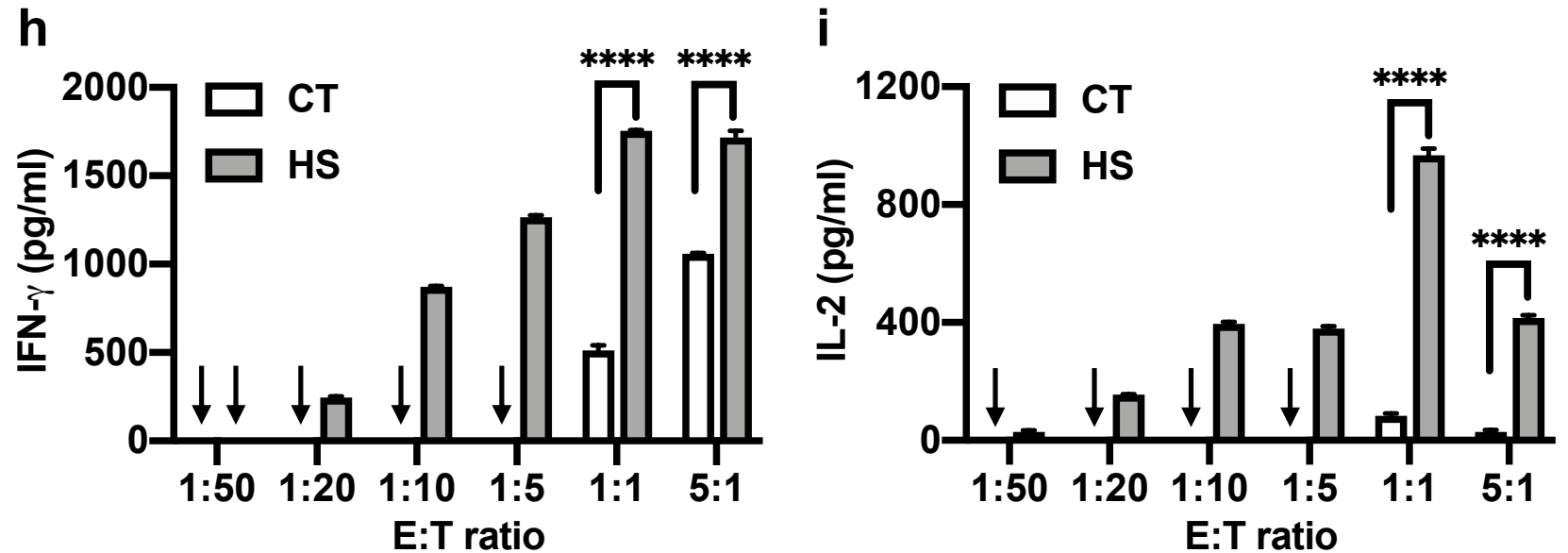


\section{Figure 3}

a

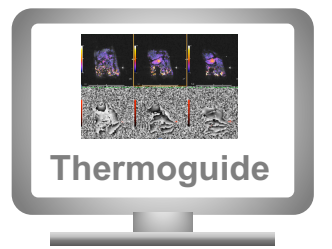

Data transfer

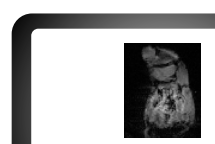

\section{MRI console}

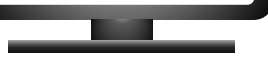

Automatic regulation
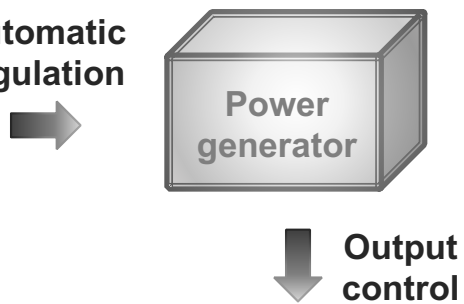
control

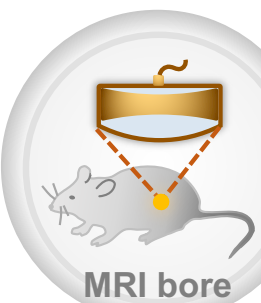

b

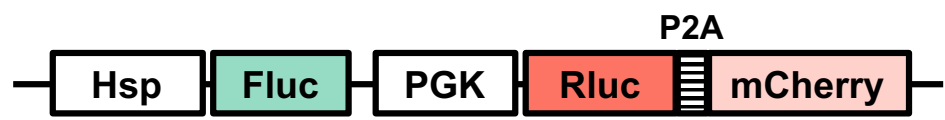

e
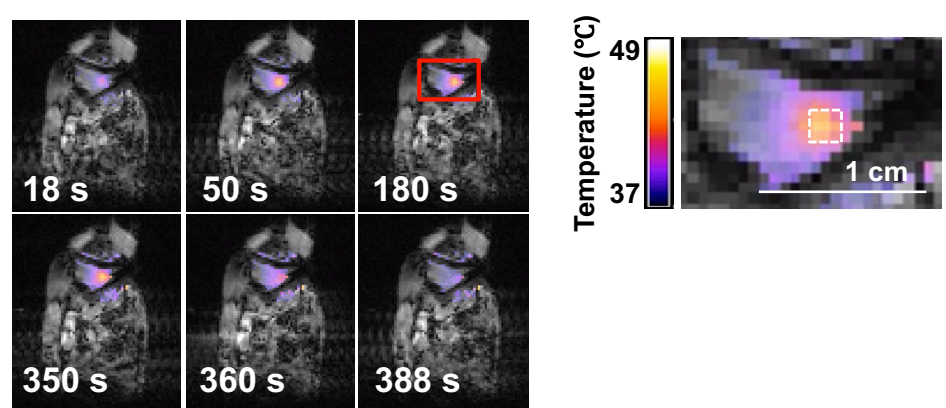

g

은

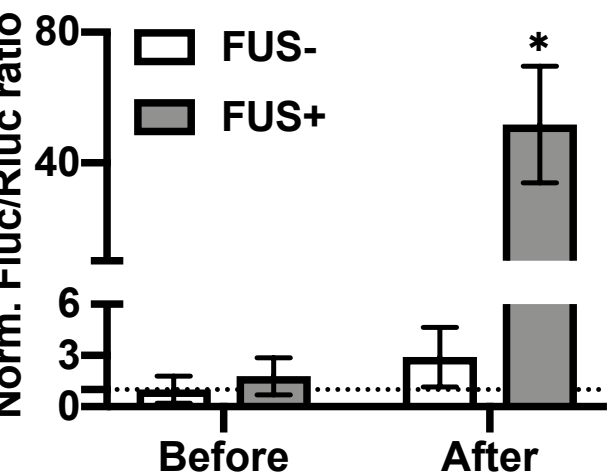

C
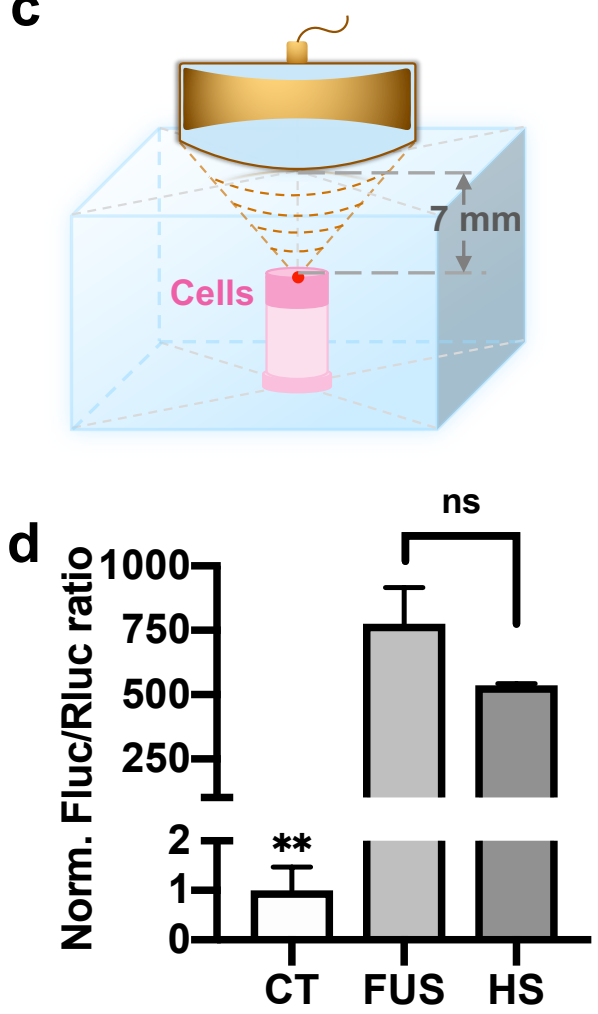

f

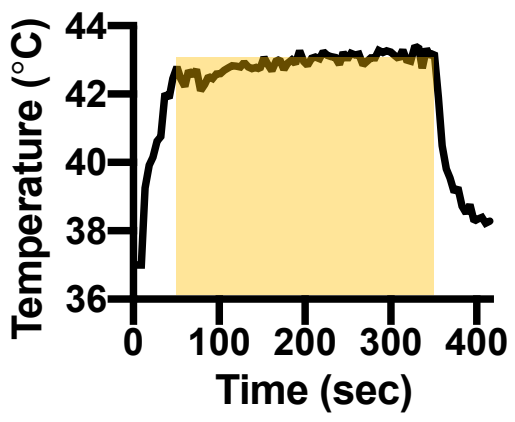

h

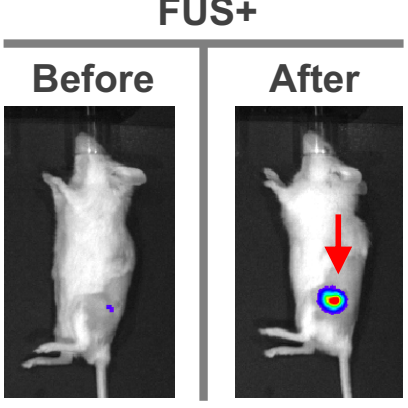




\section{Figure 4}
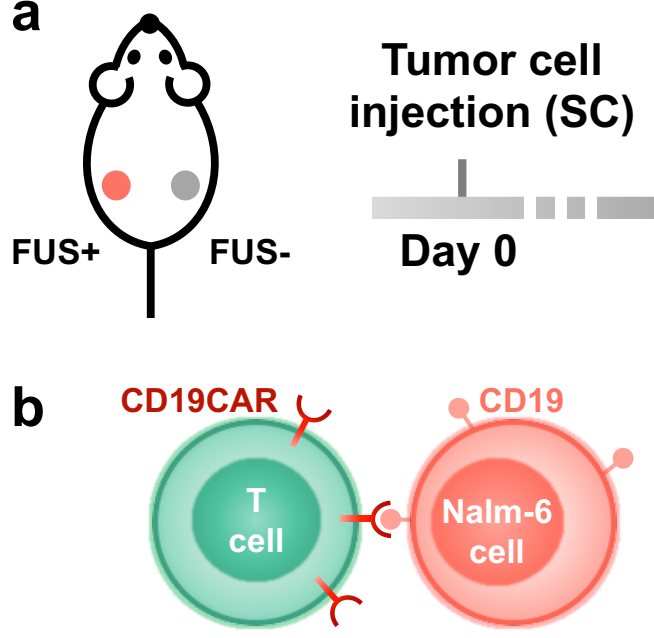

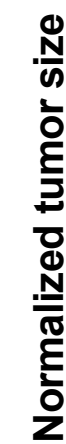

b
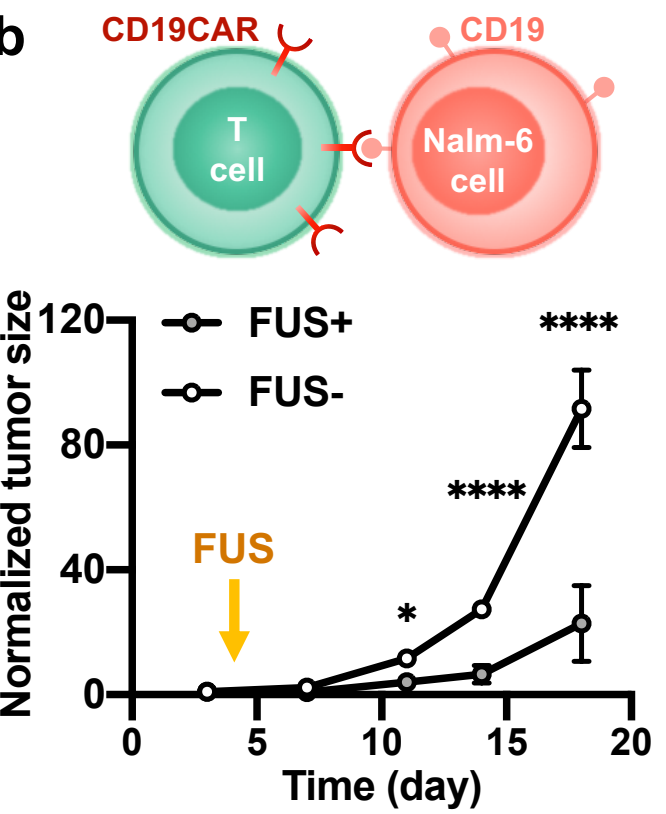

C

FUS+

FUS-

D3

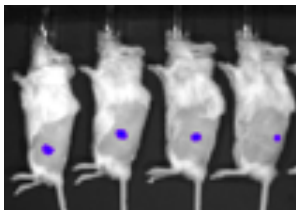

D7
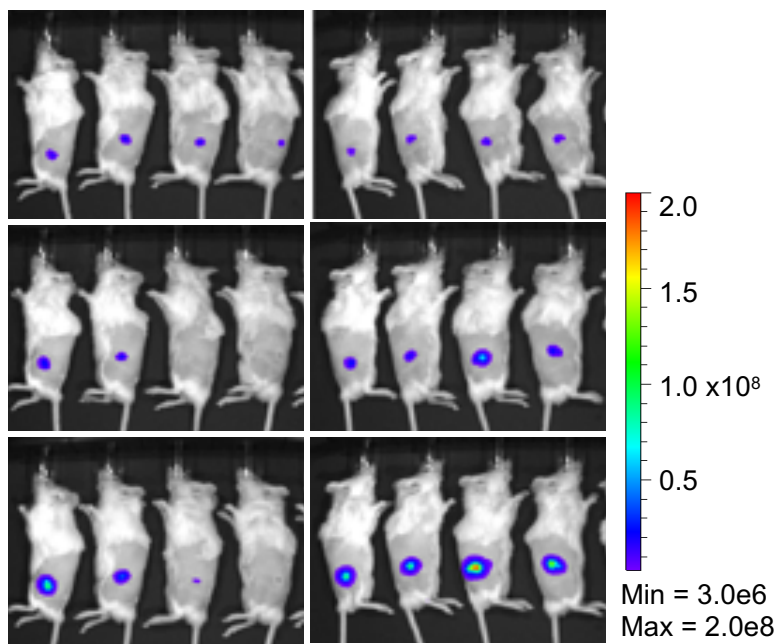

D14

D11
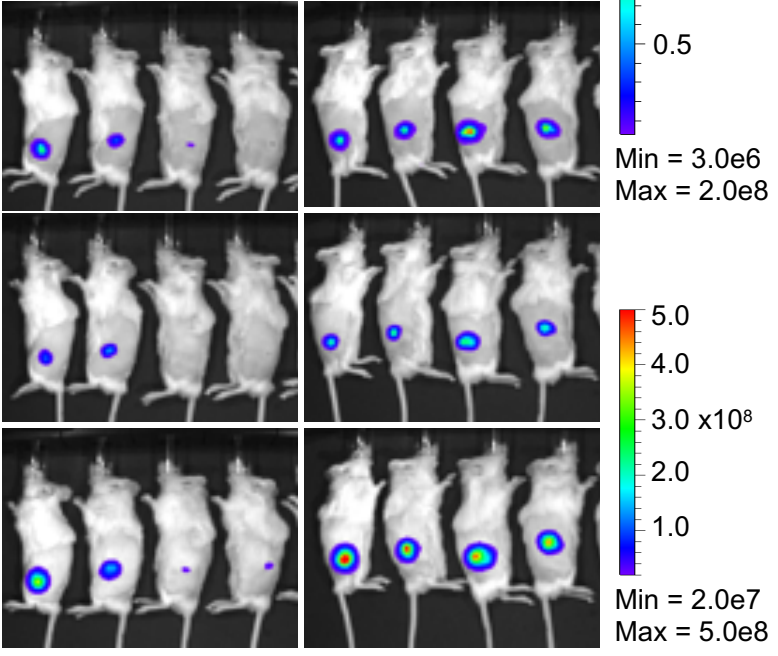
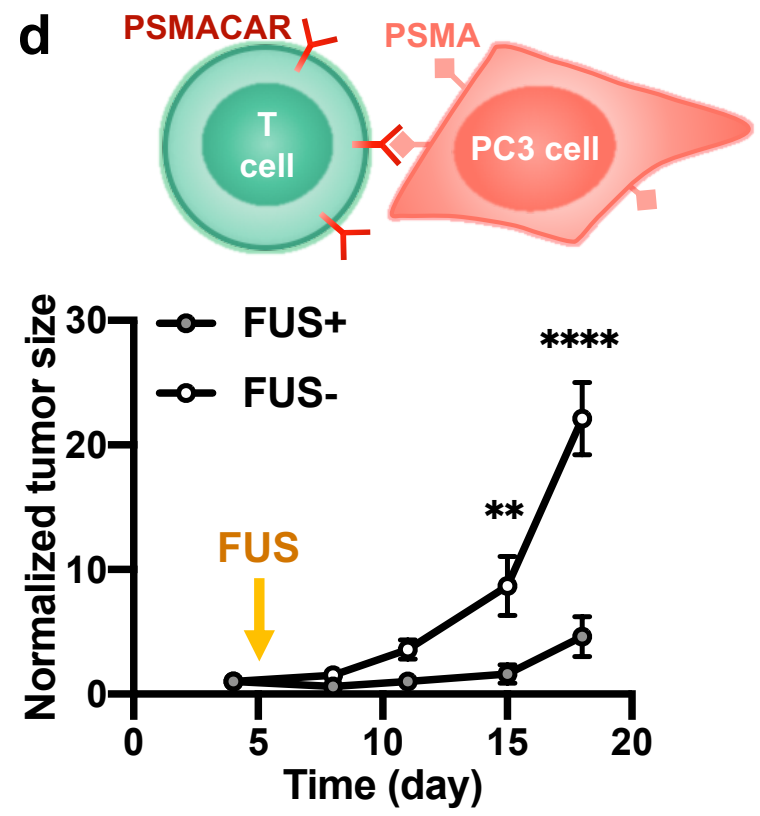

e

FUS+

FUS-

D4

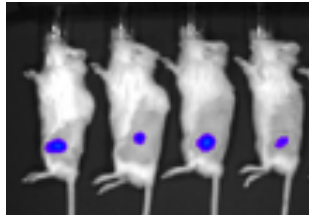

D8

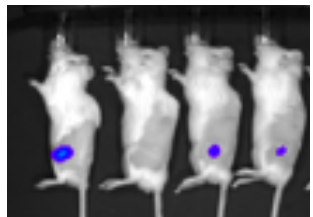

D11

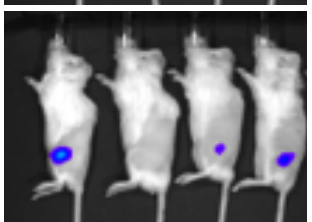

D14

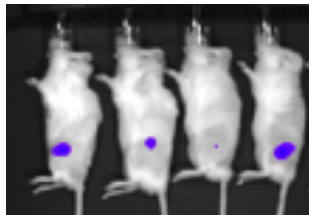

D18

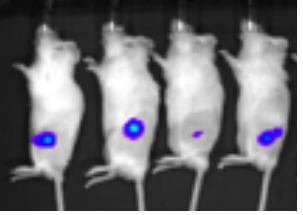

Tumor growth monitoring 